Supplement of Hydrol. Earth Syst. Sci., 21, 4131-4147, 2017

https://doi.org/10.5194/hess-21-4131-2017-supplement

(c) Author(s) 2017. This work is distributed under

the Creative Commons Attribution 3.0 License.

(c) (1)

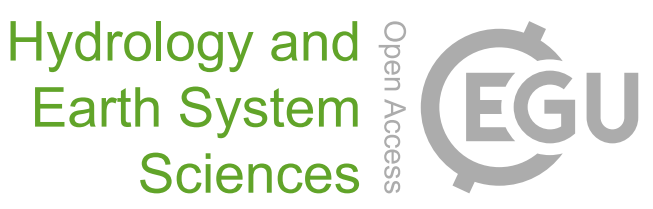

Supplement of

\title{
Comparison of the impacts of urban development and climate change on exposing European cities to pluvial flooding
}

Per Skougaard Kaspersen et al.

Correspondence to: Per Skougaard Kaspersen (pskk@dtu.dk)

The copyright of individual parts of the supplement might differ from the CC BY 3.0 License. 


\section{Supplementary materials}

\begin{tabular}{|c|c|c|c|c|c|c|c|c|}
\hline & \multicolumn{2}{|c|}{ France } & \multicolumn{2}{|c|}{ Austria } & \multicolumn{2}{|c|}{ Denmark } & \multicolumn{2}{|c|}{ France } \\
\hline & \multicolumn{2}{|c|}{ Strasbourg } & \multicolumn{2}{|c|}{ Vienna } & \multicolumn{2}{|c|}{ Odense } & \multicolumn{2}{|c|}{ Nice } \\
\hline & $\mathrm{a}$ & b & $\mathrm{a}$ & b & $\mathrm{a}$ & b & a & b \\
\hline $\begin{array}{c}\text { Linear regression } \\
\text { parameters }\end{array}$ & -246 & 135 & -244 & 124 & -245 & 135 & -321 & 151 \\
\hline
\end{tabular}

Table S1: Linear regression models used to calculate impervious surface fractions based on SAVI (from Kaspersen et al., 2015).

\begin{tabular}{|c|c|c|c|c|c|c|c|c|}
\hline & \multicolumn{2}{|c|}{ France } & \multicolumn{2}{|c|}{ Austria } & \multicolumn{2}{|c|}{ Denmark } & \multicolumn{2}{|c|}{ France } \\
\hline & \multicolumn{2}{|c|}{ Strasbourg } & \multicolumn{2}{|c|}{ Vienna } & \multicolumn{2}{|c|}{ Odense } & \multicolumn{2}{|c|}{ Nice } \\
\hline Sensor & TM & OLI & TM & OLI & TM & OLI & TM & OLI \\
\hline Path/Row & $\begin{array}{l}196 / 26 \\
196 / 26 \\
195 / 26\end{array}$ & $\begin{array}{c}196 / 26 \\
196 / 26 \\
* 196 / 26\end{array}$ & $\begin{array}{l}189 / 27 \\
190 / 26 \\
190 / 26 \\
190 / 26 \\
190 / 26\end{array}$ & $\begin{array}{l}189 / 27 \\
190 / 26 \\
190 / 26 \\
190 / 27\end{array}$ & $\begin{array}{l}195 / 21 \\
195 / 21 \\
195 / 21\end{array}$ & $\begin{array}{l}195 / 21 \\
195 / 21 \\
195 / 21 \\
195 / 22\end{array}$ & $\begin{array}{l}194 / 30 \\
194 / 30\end{array}$ & $\begin{array}{l}194 / 30 \\
194 / 30 \\
195 / 30\end{array}$ \\
\hline Date (mm/уyyy) & $\begin{array}{l}08 / 1984 \\
07 / 1985 \\
08 / 1986\end{array}$ & $\begin{array}{c}07 / 2014 \\
08 / 2015 \\
* 08 / 2015\end{array}$ & $\begin{array}{l}06 / 1984 \\
08 / 1984 \\
05 / 1986 \\
06 / 1986 \\
08 / 1986\end{array}$ & $\begin{array}{l}07 / 2013 \\
06 / 2013 \\
08 / 2013 \\
08 / 2013\end{array}$ & $\begin{array}{l}08 / 1984 \\
06 / 1986 \\
08 / 1986\end{array}$ & $\begin{array}{l}05 / 2013 \\
06 / 2013 \\
07 / 2013 \\
07 / 2013\end{array}$ & $\begin{array}{l}06 / 1984 \\
08 / 1984\end{array}$ & $\begin{array}{l}07 / 2015 \\
08 / 2015 \\
08 / 2015\end{array}$ \\
\hline
\end{tabular}

Table S2: Landsat imagery used for urban development analysis. TM = Landsat TM imagery, OLI = Landsat OLI imagery. * Both images for 196/26 from August 2015 are used. 